\title{
EFEITO DO CONSUMO DE ALIMENTO SOBRE OS VALORES ENERGÉTICOS DO GRÃO DE TRIGO E SEUS SUBPRODUTOS PARA FRANGOS DE CORTE, OBTIDOS PELA METODOLOGIA DA ALIMENTAÇÃO FORÇADA
}

\author{
Effect of the of feed intake on the energy values of the grain of wheat and its by-products for \\ broiler chickens obtained by the methodology of the forced feeding
}

\author{
Flávia Maria de Oliveira Borges ${ }^{1}$, Horácio Santiago Rostagno ${ }^{2}$, Carlos Eduardo do Prado Saad ${ }^{3}$
}

\begin{abstract}
RESUMO
Realizou-se este trabalho com aves cecectomizadas para avaliação do efeito do nível de consumo sobre os valores de energia metabolizável (energia metabolizável aparente (EMA), energia metabolizável verdadeira (EMV), energia metabolizável aparente corrigida pelo nitrogênio $\left(E M A_{n}\right)$ e energia metabolizável verdadeira corrigida pelo nitrogênio $\left.\left(E M V_{n}\right)\right)$, utilizando-se como alimentos-teste o trigo e alguns de seus produtos. O delineamento experimental foi inteiramente ao acaso, em esquema fatorial 2 x 8 (níveis de consumo x alimentos) com quatro repetições. O método utilizado para a avaliação de EM foi o da alimentação forçada. As aves foram submetidas a jejum de 30 horas e forçadas a ingerir 25 e 50 gramas do alimento puro de uma única vez. Nos alimentos e excretas, foram analisadas matéria seca, proteína bruta e energia bruta. As médias foram comparadas pelo teste de SNK. No menor nível de consumo, os valores de EMA foram menores $(\mathrm{P}<0,05)$ para todos os alimentos. A EMV do trigo integral, da farinha de trigo clara e da escura, além do farelo de trigo animal, foi maior $(\mathrm{P}<0,05)$ quando o consumo foi mais baixo. Quanto à relação EMA/EMV, ela foi maior $(\mathrm{P}<0,05)$ com o baixo consumo, explicada pelo efeito das perdas metabólica e endógena. $\mathrm{A} \mathrm{EMA}_{\mathrm{n}}$ foi afetada pelo nível de ingestão, apresentando diferenças $(\mathrm{P}<0,05)$ em todos os tratamentos devido à excreção de energia fecal metabólica mais a energia urinária endógena, as quais deprimem os valores de EMA no baixo consumo. Com relação a $\mathrm{EMV}_{\mathrm{n}}$, somente a farinha de trigo clara apresentou diferença entre tratamentos, com menor valor $(\mathrm{P}<0,05)$ no menor nível de consumo. A relação entre $\mathrm{EMA}_{\mathrm{n}}$ e $\mathrm{EMV}_{\mathrm{n}}$ foi maior $(\mathrm{P}<0,05)$ no menor nível de consumo.
\end{abstract}

Termos para indexação: Aves, alimentação forçada, nível de consumo, energia metabolizável.

\begin{abstract}
This paper were carried out with caecectomised broilers for evaluation of the effects of the feed intake levels on the methodology of forced feeding, for evaluation of the metabolizable energy (apparent metabolizable energy - AME, true metabolizable energy - TME, apparent corrected by the nitrogen metabolizable energy - $\mathrm{AME}_{\mathrm{n}}$ and true corrected - $\mathrm{TME}_{\mathrm{n}}$ ), using as feeds tests the wheat and some of its by-products randomly distributed in fatorial outline of 2 x 8 , (levels $x$ feeds) with four repetitions. The method used for the evaluation of ME was the forced feeding. All the broilers were submitted to a fast of 30 hours and forced to ingest 25 and 50 grams of the pure feeds of an only time. In the two levels a treatment was left in fast, for the determination of the metabolic losses and endogenous. In the feeds and excrete, dry matter, crude protein and gross energy they were analyzed. The averages were compared by the test of Student Newman Keuls. Evaluated the effects of the consumption levels on the metabolizable energy obtained by the methodology of Sibbald, it was observed that the values of AME for over all treatments were affected for the amount of ingested feed, and in the lowest consumption level the values of AME were significantly lower. Wheat bran and the by-products values obtained for TME were higher in the level of lower consumption. With regard to the relationship AME/TME, would be logical to wait larger relationships at low consumption levels, as well as it was found, once the effects of the metabolic and endogenous losses present larger influences in the calculation of TME in low ingestions. $\mathrm{AME}_{\mathrm{n}}$ was affected strongly by the ingestion levels, presenting significant differences in all the treatments. These results are due to the excretion of metabolic fecal energy (FEm) more the energy endogenous urinary (EUe) that depresses the values of EMA in low consumptions. With relationship $\mathrm{TME}_{\mathrm{n}}$, only the clear wheat flour shown difference among the feeds tested, with lowest value of $\mathrm{TME}_{\mathrm{n}}$ in the smallest consumption level. The other treatments did not show any significant differences. The relationship between $\mathrm{AME}_{\mathrm{n}}$ and $\mathrm{TME}_{\mathrm{n}}$ was larger in the smallest consumption levels.
\end{abstract}

Index terms: Birds, forced feeding, consumption levels, metabolizable energy.

(Recebido para publicação em 6 de abril de 2004 e aprovado em 19 de outubro de 2004)

1. Médica Veterinária - M.Sc., Dra. em Nutrição Animal - Universidade Federal de Lavras/UFLA - Caixa Postal 3037 - $37200-000$ - Lavras, MG. borges@ufla.br
2. Engenheiro Agrônomo, M.Sc., PhD Nutrição Animal, Universidade Federal de Viçosa - Departamento de Zootecnia - $36570-000$ - Viçosa, MG.

3. Zootecnista, MSc., Dr. Nutrição Animal, Fundação Zoobotânica de Belo Horizonte. 


\section{INTRODUÇÃO}

A formulação de dietas com concentração variável de energia disponível deve levar em consideração o aporte dos outros nutrientes. Segundo Albino (1991), nos últimos anos, foram desenvolvidos vários métodos diretos, os quais geraram diversas definições para expressar os valores de energia metabolizável (EM) dos alimentos para aves. Os mais utilizados são os ensaios realizados segundo a metodologia tradicional, nos quais se obtêm a EM aparente (EMA), e os ensaios com alimentação forçada, nos quais se obtém a EM verdadeira (EMV).

Em razão das limitações da metodologia tradicional, Sibbald (1976) sugeriu uma técnica baseada na metodologia de alimentação forçada, que consiste em alimentar forçosamente as aves com uma quantidade conhecida de alimento, controlando, dessa forma, o fator que mais influencia os resultados o nível de consumo. Segundo Sibbald (1975), a EMA pode variar com o nível de ingestão porque, sob condições padronizadas, a excreção de energia fecal metabólica mais a urinária endógena é constante. Quando o nível de consumo é alto, a influência das perdas metabólicas é pequena; entretanto, quando o consumo é baixo, essas perdas podem diminuir consideravelmente a EMA. O método proposto por Sibbald (1976) considerava as perdas fecais metabólicas e urinárias endógenas e assumia que as frações endógena e metabólica que compõem as excretas das aves alimentadas são as mesmas que as das aves em jejum. O maior interesse por essa metodologia, no entanto, tem sido a rapidez nas determinações e por ser menos oneroso, quando comparado ao sistema normal usado de EMA (DEVEGOWDA et al., 1986; ALBINO et al., 1992).

A metodologia da alimentação forçada apresenta uma série de críticas - uma delas é a de que as aves que receberam pequena quantidade de alimento poderiam catabolizar maior quantidade de proteína corporal do que as aves que receberam maior quantidade de alimentos. Nesse caso, o catabolismo protéico seria máximo nas aves em jejum e se reduziria com o aumento de ingestão de alimento. Esse fato levaria a erros na correção das perdas metabólica e endógena, uma vez que o aumento de excreção protéica superestimaria o nitrogênio e a energia das aves em jejum, subestimando os valores de EMV e energia metabolizável verdadeira corrigida $\left(\mathrm{EMV}_{\mathrm{n}}\right)$.

É evidente que o erro experimental induzido pela maior excreção endógena de aves em jejum, quando comparadas com aquelas que receberam alimentação à vontade, seria maior à medida que o consumo de alimento fosse diminuído. Além disso, quantidade reduzi- da de alimento resultaria em menor excreção, o que poderia per si elevar o erro experimental. Experimentos com níveis de ingestão de 50 gramas apresentaram resultados mais consistentes, baseados nas seguintes questões: as excreções endógena e metabólica das aves constituem parte significativa da excreta das aves que receberam pequena quantidade de alimento, e essa excreção pode se apresentar distinta daquela obtida em aves em jejum, além do que pequena quantidade de alimento pode aumentar o erro experimental (SIBBALD, 1977). O autor reconhece que o efeito do erro experimental pode ser inversamente proporcional à quantidade de alimento administrada; entretanto, cita que níveis acima de 40 gramas por ave podem provocar regurgitação, que tende a aumentar em incidência e severidade com o aumento da quantidade de alimento. A recomendação do autor é de que se utilize uma quantidade de alimentos entre 25 e 50 gramas, sendo o nível ótimo de alimentação forçada de aproximadamente $40 \mathrm{~g}$, quando o ensaio é realizado com aves adultas.

A outra questão seria com respeito à natureza do alimento oferecido. Alimentos mais fibrosos tendem a aumentar as excreções metabólica e endógena, e os efeitos dessa excreção seriam mais intensos quando pequenas quantidades de alimentos são oferecidas. Entretanto, Sibbald (1980) argumenta que mudanças induzidas pela natureza ou quantidade de fibra dietética são insignificantes.

Com este trabalho teve-se por objetivo avaliar o efeito do nível de consumo de alimento sobre a energia metabolizável aparente (EMA), metabolizável verdadeira (EMV), metabolizável aparente corrigida pelo nitrogênio $\left(\mathrm{EMA}_{\mathrm{n}}\right)$ e metabolizável verdadeira corrigida pelo nitrogênio $\left(E M V_{n}\right)$ em frangos de corte avaliados pela metodologia de alimentação forçada.

\section{MATERIAL E MÉTODOS}

Neste trabalho, foi comparado o efeito do nível de consumo de alimento sobre o valor da EM obtido pela metodologia de alimentação forçada (SIBBALD, 1976). Foram comparados dois níveis de consumo (25 e 50 gramas) utilizando-se uma ração-referência e sete alimentos-teste, constituídos de produtos oriundos do trigo. Foram utilizados em todos os métodos: trigo integral moído (TIM), gérmen de trigo (GT), farinha de trigo clara (FTC), farinha de trigo escura (FTE), farelo de trigo para uso humano (FTH), farelo de trigo para uso animal (FTA) e farelo de trigo grosso (FTG). A dietareferência constituiu um tratamento e foi calculada segundo recomendações de Rostagno et al. (1985), à base de milho moído, farelo de soja, óleo de soja e mistura vitamínico-mineral (Tabela 1 ). 
Os dados experimentais do nível de $25 \mathrm{~g}$ foram obtidos utilizando 36 aves previamente cecectomizadas, segundo Pupa et al. (1998). Trinta e duas aves foram distribuídas em oito tratamentos e quatro repetições, com uma ave por unidade experimental, utilizando-se o método da alimentação forçada descrito por Sibbald (1976). Um tratamento - 9 (uma ave por repetição x quatro repetições = quatro aves) foi utilizado para estimar as perdas endógenas e metabólicas. Esse tratamento não fez parte das análises estatísticas.

O período experimental foi de quatro dias, obedecendo ao seguinte cronograma: todas as aves foram submetidas ao jejum de 30 horas para esvaziamento completo do trato gastrintestinal e, após o jejum foram, forçadas a ingerir 25 gramas dos alimentos-teste de uma única vez. As do tratamento 9 foram deixadas em jejum por mais 48 horas para determinação das perdas metabólicas e endógenas. As aves tiveram toda a região da cloaca previamente depenada para evitar aderência da excreta.

A coleta de excreta foi total, de 12 em 12 horas, até completar o período exato de 48 horas, para todas as aves, inclusive o jejum, e as excretas foram estocadas congeladas $\left(-8^{\circ} \mathrm{C}\right)$ até o final do experimento, quando foram agrupadas por repetição e homogeneizadas para futuras análises.

TABELA 1 - Composição da ração-referência.

\begin{tabular}{|c|c|c|c|}
\hline Ingredientes & & kg & \\
\hline Milho moído & & 52,000 & \\
\hline Farelo de soja & & 41,867 & \\
\hline Óleo de soja & & 2,533 & \\
\hline Suplemento vitamínico e mineral ${ }^{2}$ & & 0,600 & \\
\hline Fosfato bicálcio & & 2,400 & \\
\hline DL Metionina & & 0,200 & \\
\hline Sal & & 0,400 & \\
\hline Total & & 100,00 & \\
\hline Composição Calculada $^{1}$ & Matéria natural (\%) & & Matéria Seca (\%) \\
\hline Proteína bruta \% & 23,00 & & 25,84 \\
\hline $\mathrm{EM} \mathrm{kcal} / \mathrm{kg}$ & 2930 & & 3300 \\
\hline Cálcio Total \% & 1,00 & & 1,12 \\
\hline Fósforo disponível & 0,57 & & 0,64 \\
\hline Fósforo total & 0,83 & & 0,92 \\
\hline
\end{tabular}

1- Valores calculados segundo Rostagno et al. (1985)

2- Formula da fábrica de rações Itambé: Bacitracina de zinco (10\%) - 6000 mg; coccidiostático (salinomicina) - 50 gramas; Vitamina $B_{6}(98 \%)$ - 1240 mg; Vitamina $B_{12}(0,1 \%)$-1200 mg; Vitamina $B_{2}(96 \%)$ - 420 mg; Vitamina A (500.000 UI/g) - 2000 mg, Vitamina $D_{3}(500000$ UI/g) - 260 mg; Pantotenato de Cálcio (98\%) - 1340 mg; Vitamina E (50\%) - 2400 mg; Vitamina $K_{3}$ (52\%) -3880 mg; Vitamina B (98\%) - 640 mg; Biotina (2\%) 1000 mg; Vitamina C (97,5\%) - 5140 mg; Ácido Fólico (98\%) - 76 mg; Ácido Nicotínico (100\%) - 2800 mg, Antioxidante - 15 g; Selenito de Sódio (45\%) - 32 mg; Iodato de Cálcio (62\%) - 100 mg, Sulfato de Cobre (25\%) - 4060 mg; Sulfato de Manganês (28\%) - 25,72 g; sulfato Ferroso (28\%) - 34,7 g; Óxido de Zinco (75\%) - 11,82 g; Cloreto de Colina - 41,664 g; Veículo - 373,996 g. 
Para o consumo de $50 \mathrm{~g}$, utilizaram-se as mesmas 36 aves, sendo 32 para obtenção dos dados de EM e quatro para obtenção das perdas fecal e metabólica.

As análises bromatológicas e de energia foram realizadas no laboratório de nutrição animal da Escola de Veterinária da UFMG, adotando-se os seguintes procedimentos para as amostras de alimentos puros, ração-referência e excretas: matéria seca (MS), determinada em estufa de $105^{\circ} \mathrm{C}$ e $55^{\circ} \mathrm{C}$, segundo Cunniff (1995); nitrogênio total ou proteína bruta (PB), determinada pelo método de Kjeldahl, segundo Cunniff (1995); energia bruta (EB), utilizando-se calorímetro adiabático PARR.

Para a avaliação dos efeitos do nível de consumo pela a metodologia de alimentação forçada, o delineamento experimental foi inteiramente ao acaso, em esquema fatorial $2 \times 8$, (nível de consumo x alimentos) com 4 repetições. As médias dos tratamentos foram comparadas pelo teste SNK. As metodologias para os cálculos de EM foram semelhantes às descritas por Albino (1991).

\section{RESULTADOS E DISCUSSÃO}

Os resultados da EMA e EMV encontram-se na Tabela 2 e ocorreram diferenças $(P<0,05)$ entre os dois níveis de consumo. Para a EMA, ocorreu uma interação significativa, pelo teste de $\mathrm{F}(\mathrm{P}<0,01)$, entre níveis e tipos de alimentos; entretanto, essa interação não foi observada para a EMV. Os valores de EMA para todos os alimentos foram superiores no nível de maior consumo, o que já era esperado, uma vez que nesse nível o volume de excreta é maior, minimizando a influência causada pelas perdas metabólica e endógena.

Contrário ao que afirmam Sibbald (1979) e Sibbald e Morse (1982), alguns alimentos apresentaram diferenças significativas entre níveis quanto aos valores de EMV. Nos alimentos TIM, FTC e FTA, os valores da EMV foram maiores no nível de consumo mais baixo, e o valor de FTE foi maior no consumo de 50 gramas. Os valores de EMV dos demais alimentos não apresentaram diferenças entre níveis de consumo. Esses resultados assemelham-se aos de Pesti (1984), que sugere que a variação no nível de ingestão pode alterar os valores de EMV, uma vez que com baixo consumo pode ocorrer mobilização de tecidos, que modifica a quantidade de nitrogênio excretada. Flores e Castanon (1991) acrescentam que baixo nível de consumo poderia aumentar o erro experimental.
Os resultados deste trabalho são semelhantes aos encontrados por Sibbald (1977). O autor sugere que o nível ótimo de alimentação forçada situa-se em aproximadamente $40 \mathrm{~g}$, quando o ensaio é realizado com aves adultas. As quantidades usadas pelo autor variaram de 10 a 100 gramas, com aumentos de 10 em 10 gramas. Acima de 40 gramas por ave, ocorreu regurgitação, a qual tendeu a aumentar em incidência e severidade com $\mathrm{o}$ aumento da quantidade de alimento. $\mathrm{O}$ desvio-padrão da média da EMV diminuiu com o aumento de ingestão até 30 gramas. Acima de 60 g o número de dados experimentais foi reduzido, porque o alimento regurgitado contaminou a excreta.

TABELA 2 - Valores de energia metabolizável aparente e verdadeira ( $\mathrm{kcal} / \mathrm{kg}$ de MS) segundo o alimento ingerido.

\begin{tabular}{lcccc}
\hline \multirow{2}{*}{$\begin{array}{c}\text { Alimen- } \\
\text { to }\end{array}$} & \multicolumn{2}{c}{ EMA } & \multicolumn{2}{c}{ EMV } \\
\cline { 2 - 5 } & $\mathbf{( 2 5} \mathbf{g})$ & $\mathbf{( 5 0} \mathbf{g})$ & $\mathbf{( 2 5} \mathbf{g})$ & $\mathbf{( 5 0} \mathbf{g})$ \\
\hline RR & $2170 \mathrm{~b}$ & $2704 \mathrm{a}$ & 3388 & 3371 \\
TIM & $2932 \mathrm{~b}$ & $3330 \mathrm{a}$ & $4179 \mathrm{a}$ & $3983 \mathrm{~b}$ \\
GT & $1991 \mathrm{~b}$ & $2627 \mathrm{a}$ & 3206 & 3292 \\
FTC & $2783 \mathrm{~b}$ & $3203 \mathrm{a}$ & $4022 \mathrm{a}$ & $3881 \mathrm{~b}$ \\
FTE & $2527 \mathrm{~b}$ & $3243 \mathrm{a}$ & $3753 \mathrm{~b}$ & $3915 \mathrm{a}$ \\
FTH & $1013 \mathrm{~b}$ & $1510 \mathrm{a}$ & 2223 & 2173 \\
FTA & $1250 \mathrm{~b}$ & $1626 \mathrm{a}$ & $2459 \mathrm{a}$ & $2289 \mathrm{~b}$ \\
FTG & $1170 \mathrm{~b}$ & $1677 \mathrm{a}$ & 2400 & 2351 \\
Média & 1980 & 2490 & 3204 & 3157 \\
CV & 3,10 & 2,26 & 1,91 & 1,78 \\
\hline
\end{tabular}

Valores na mesma linha, para a mesma variável, seguidos de letras distintas diferem entre si, pelo teste de $F(P<0,05)$

1: $\mathrm{RR}=$ ração-referência, TIM = trigo integral moído, GT=germe de trigo, FTC = farinha de trigo clara, FTE = farinha de trigo escura, FTH = farelo de trigo para uso humano, FTA $=$ farelo de trigo para uso animal, FTG = farelo de trigo grosso.

Do mesmo modo que para EMA, ocorreu uma interação significativa, pelo teste de $\mathrm{F}(\mathrm{P}<0,01)$, entre níveis e tipos de alimentos para a EMAn. Igualmente à EMV, essa interação não foi observada para a EMVn. A 
$\mathrm{EMA}_{\mathrm{n}}$ foi afetada $(\mathrm{P}<0,05)$ pelos níveis de ingestão, apresentando diferenças significativas entre os tratamentos, para todos os alimentos (Tabela 3). Embora a correção pelo nitrogênio amenize as diferenças causadas pelas excreções metabólica e endógena, essa correção per si não foi suficiente para eliminar o efeito das perdas sobre o baixo consumo.

Com relação à EMV $\mathrm{V}_{\mathrm{n}}$ (Tabela 3), somente a farinha de trigo clara apresentou diferença entre tratamentos, com menor valor no consumo de 25 gramas. Os outros alimentos não apresentaram diferenças entre os níveis de ingestão. Esses resultados mostram que a EMV corrigida pelo nitrogênio é a medida energética menos afetada pelo nível de consumo, e parece ser a melhor forma de estimar o conteúdo energético dos alimentos para aves.

Segundo Dale e Fuller (1984), o balanço de nitrogênio (BN) é o nitrogênio ingerido menos o nitrogênio excretado, sendo esse último de origem alimentar, fecal metabólico e urinário endógeno. Assim, o nitrogênio excretado deve ser corrigido para que reflita somente o nitrogênio de origem alimentar, e a correção possa ser feita com base no nitrogênio retido. Essa correção é feita considerando a excreta de aves em jejum; entretanto, pode apresentar falhas, uma vez que as aves em jejum podem catabolizar proteínas de tecidos para manutenção da gliconeogênese, aumentando a excreção de nitrogênio. Isso poderia ocorrer com aves que receberam pequena quantidade de alimento e não ocorreria com aves que consumiram grande quantidade da dieta. Os resultados obtidos neste trabalho confirmam essas afirmações, uma vez que as diferenças provocadas pelo consumo de alimento foram maiores ao se avaliar a EMV, e menores ou não existentes na $\mathrm{EMV}_{\mathrm{n}}$. Segundo Dale e Fuller (1986), o BN pode reduzir erros de variância na estimativa de energia metabolizável em até $40 \%$, dependendo do tipo de alimento.

Com os resultados deste trabalho, também se reforçam os encontrados por Wolynetz e Sibbald (1984), que discutem sobre algumas relações entre as quatro estimativas comuns de energia biodisponível (Edisp): EMA, EMV e seus equivalentes corrigidos pelo balanço de nitrogênio, $\mathrm{EMA}_{\mathrm{n}}$ e $E M V_{\mathrm{n}}$. Tanto a EMA quanto $\mathrm{EMA}_{\mathrm{n}}$ subestimam a Edisp, principalmente no baixo nível de ingestão. Dentro de níveis de ingestão à vontade, a EMA é menor do que a $\mathrm{EMA}_{\mathrm{n}}$ quando o nitrogênio retido era menor que zero e a EMA é maior que a $\mathrm{EMA}_{\mathrm{n}}$ quando o $\mathrm{BN}$ era maior que zero. Em baixo nível de consumo, a EMV tende a superestimar a Edisp, mas essa diferença é menor que a subestimativa produzida pela EMA ou $\mathrm{EMA}_{\mathrm{n}}$. A $\mathrm{EMV}_{\mathrm{n}}$ apresenta uma boa estimativa da Edisp em todos os níveis de consumo. A precisão dos estimadores da Edisp aumenta com o incremento do nível de ingestão.

Na Tabela 4 encontram-se os valores de nitrogênio excretado em porcentagem de excreta e o balanço de nitrogênio dos alimentos. As aves que consumiram menor quantidade de alimento apresentaram maior valor de N na excreta, com exceção do GT, FTA e FTG. Esses resultados são semelhantes aos de Pesti (1984), que sugere que pequena quantidade de alimento consumido pode causar mobilização de tecidos, o que modificaria a quantidade de nitrogênio excretada.

TABELA 3 - Valores de energia metabolizável aparente e verdadeira corrigidas ( $\mathrm{kcal} / \mathrm{kg}$ de MS) segundo o alimento ingerido.

\begin{tabular}{lcccc}
\hline \multirow{2}{*}{$\begin{array}{c}\text { Alimen- } \\
\mathbf{t o}^{\mathbf{1}}\end{array}$} & \multicolumn{2}{c}{$\mathbf{E M A}_{\mathbf{n}}$} & \multicolumn{2}{c}{$\mathbf{E M V}_{\mathbf{n}}$} \\
\cline { 2 - 5 } & $\mathbf{( 2 5} \mathbf{g})$ & $\mathbf{( 5 0} \mathbf{g})$ & $\mathbf{( 2 5} \mathbf{g})$ & $\mathbf{( 5 0} \mathbf{g})$ \\
\hline RR & $1785 \mathrm{~b}$ & $2433 \mathrm{a}$ & 3004 & 3100 \\
TIM & $2728 \mathrm{~b}$ & $3155 \mathrm{a}$ & 3976 & 3838 \\
GT & $1620 \mathrm{~b}$ & $2310 \mathrm{a}$ & 2835 & 2976 \\
FTC & $2683 \mathrm{~b}$ & $3022 \mathrm{a}$ & $3923 \mathrm{a}$ & $3701 \mathrm{~b}$ \\
FTE & $2329 \mathrm{~b}$ & $3014 \mathrm{a}$ & 3556 & 3686 \\
FTH & $710 \mathrm{~b}$ & $1295 \mathrm{a}$ & 1921 & 1958 \\
FTA & $718 \mathrm{~b}$ & $1462 \mathrm{a}$ & 1927 & 2125 \\
FTG & $863 \mathrm{~b}$ & $1522 \mathrm{a}$ & 2093 & 2196 \\
Média & 1680 & 2277 & 2779 & 2948 \\
CV & 8,00 & 3,87 & 4,63 & 3,00 \\
\hline
\end{tabular}

Valores na mesma linha, para a mesma variável, seguidos de letras distintas diferem entre si, pelo teste de F $(P<0,05)$

1: $\mathrm{RR}=$ ração-referência, TIM = trigo integral moído, GT=germe de trigo, FTC = farinha de trigo clara, FTE = farinha de trigo escura, FTH = farelo de trigo para uso humano, FTA = farelo de trigo para uso animal, FTG = farelo de trigo grosso. 
TABELA 4 - Valores de nitrogênio excretado ${ }^{1}$ e balanço de nitrogênio ${ }^{2}$ segundo o alimento ingerido.

\begin{tabular}{lccccc}
\hline \multirow{2}{*}{ Alimento $^{3}$} & \multirow{2}{*}{ N alimento (\% MS) $^{2}$} & \multicolumn{2}{c}{$\mathbf{N}$ excretado (g/100g de excreta) } & \multicolumn{2}{c}{ Balanço de Nitrogênio } \\
\cline { 3 - 6 } RR & $\mathbf{( 2 5} \mathbf{g})$ & $\mathbf{( 5 0} \mathbf{g})$ & $\mathbf{( 2 5} \mathbf{g})$ & $\mathbf{( 5 0} \mathbf{g})$ \\
TIM & 2,12 & $13,01 \mathrm{a}$ & $11,12 \mathrm{~b}$ & $1,04 \mathrm{~b}$ & $1,47 \mathrm{a}$ \\
GT & 2,51 & $15,69 \mathrm{a}$ & $11,58 \mathrm{~b}$ & $0,54 \mathrm{~b}$ & $0,97 \mathrm{a}$ \\
FTC & 5,12 & 14,44 & 12,42 & $1,01 \mathrm{~b}$ & $1,71 \mathrm{a}$ \\
FTE & 2,26 & $18,25 \mathrm{a}$ & $11,11 \mathrm{~b}$ & $0,27 \mathrm{~b}$ & $0,95 \mathrm{a}$ \\
FTH & 2,93 & $15,99 \mathrm{a}$ & $13,61 \mathrm{~b}$ & 0,54 & 1,22 \\
FTA & 3,14 & $9,37 \mathrm{a}$ & $7,35 \mathrm{~b}$ & 0,96 & 1,17 \\
FTG & 2,89 & 5,76 & 7,50 & 1,58 & 0,89 \\
Média & 2,96 & 9,33 & 7,38 & 0,90 & 0,83 \\
R & & 12,73 & 10,26 & 0,86 & 1,15 \\
CV & & 0,83 & 0,69 & 0,61 & 0,42 \\
\hline
\end{tabular}

Valores na mesma linha, para a mesma variável, seguidos de letras distintas diferem entre si, pelo teste de $F$ $(\mathbf{P}<0,05)$

1: Valores expressos em g de $\mathrm{N} / 100 \mathrm{~g}$ de MS de excreta

2: Obtido pela fórmula $-\mathrm{BN}=\mathrm{N}$ consumido $-(\mathrm{N}$ excretado $-\mathrm{N}$ endógeno e metabólico)

3: $\mathrm{RR}=$ ração-referência, $\mathrm{TIM}=$ trigo integral moído, GT=germe de trigo, FTC $=$ farinha de trigo clara, FTE = farinha de trigo escura, FTH = farelo de trigo para uso humano, FTA = farelo de trigo para uso animal, FTG = farelo de trigo grosso.

Todas as aves apresentaram balanço positivo de nitrogênio, tanto para o consumo de 25 gramas como para o de 50 gramas. Seria de se esperar que aves nessas circunstâncias, ou seja, recebendo quantidade de alimento menor que o exigido para manutenção, perdessem peso e, conseqüentemente apresentassem balanço negativo de $\mathrm{N}$. A explicação possível é que o tempo experimental foi curto o suficiente para evitar que a perda de peso refletisse no BN.

Comparando os dois níveis de ingestão, pode-se observar que mesmo em balanço positivo de $\mathrm{N}$, as aves com o consumo de $25 \mathrm{~g}$ apresentaram menores valores de BN quando receberam os alimentos RR, GT, TIM e FTC, o que significa que essas aves apresentaram, proporcionalmente, maior excreção de $\mathrm{N}$.

As relações entre EMV/EMA e $\mathrm{EMV}_{\mathrm{n}} / \mathrm{EMA}_{\mathrm{n}}$ encontram-se nas Tabelas 5 e 6, respectivamente. Essa relação foi estabelecida porque Sibbald (1977) propõe a utilização de fatores de correção para estimar a EMV dos alimentos a partir da EMA ou viceversa.

No menor nível de consumo, os valores da relação EMV/EMA (Tabela 5) e $\mathrm{EMV}_{\mathrm{n}} / \mathrm{EMA}_{\mathrm{n}}$ (Tabela 6) foram maiores para todos os alimentos $(P<0,05)$. Isso demonstra que existe uma grande interferência das perdas endógena e metabólica na EMA e essa interferência é maior no menor nível de consumo. Além disso, os valores dessas relações foram menores naqueles alimentos cuja energia metabolizável foi mais alta. Os farelos apresentaram os maiores valores paras essas relações tanto no consumo de 25 gramas como no de 50 gramas. Dessa forma, a utilização de fatores de transformação de $E M V / E M A$ ou $E M V_{n} / E M A_{n}$ não é válida, uma vez que esses fatores podem variar largamente em função da quantidade de alimento consumido ou mesmo em função do tipo de alimento testado. 
TABELA 5 - Relação entre energia metabolizável aparente e verdadeira segundo o alimento ingerido.

\begin{tabular}{lcc}
\hline \multirow{2}{*}{ Alimentos $^{\mathbf{1}}$} & \multicolumn{2}{c}{ Relação EMV/EMA } \\
\cline { 2 - 3 } & $\mathbf{( 2 5 g )}$ & $\mathbf{( 5 0 g )}$ \\
\hline RR & $1,561 \mathrm{a}$ & $1,247 \mathrm{~b}$ \\
TIM & $1,426 \mathrm{a}$ & $1,207 \mathrm{~b}$ \\
GT & $1,610 \mathrm{a}$ & $1,253 \mathrm{~b}$ \\
FTC & $1,445 \mathrm{a}$ & $1,211 \mathrm{~b}$ \\
FTE & $1,486 \mathrm{a}$ & $1,207 \mathrm{~b}$ \\
FTH & $2,199 \mathrm{a}$ & $1,439 \mathrm{~b}$ \\
FTA & $1,969 \mathrm{a}$ & $1,407 \mathrm{~b}$ \\
FTG & $1,993 \mathrm{a}$ & $1,401 \mathrm{~b}$ \\
Média & 1,711 & 1,297 \\
CV & 3,98 & 0,63 \\
R & 0,956 & 0,994 \\
\hline
\end{tabular}

Valores na mesma linha, seguidos de letras distintas, diferem entre si, pelo teste de $F(P<0,05)$

1: $\mathbf{R R}=$ ração-referência, $\mathbf{T I M}=$ trigo integral moído, GT=germe de trigo, $\mathrm{FTC}=$ farinha de trigo clara, $\mathrm{FTE}=$ farinha de trigo escura, $\mathrm{FTH}=$ farelo de trigo para uso humano, FTA = farelo de trigo para uso animal, FTG = farelo de trigo grosso.

TABELA 6 - Relação entre energia metabolizável aparente corrigida e verdadeira corrigida segundo o alimento ingerido.

\begin{tabular}{|c|c|c|}
\hline \multirow{2}{*}{ Alimento $^{1}$} & \multicolumn{2}{|c|}{ Relação EMV } \\
\hline & (25g) & (50g) \\
\hline $\mathrm{RR}$ & 1,683a & $1,274 b$ \\
\hline TIM & $1,457 \mathrm{a}$ & $1,217 b$ \\
\hline GT & $1,753 a$ & $1,288 b$ \\
\hline FTC & $1,462 \mathrm{a}$ & $1,225 b$ \\
\hline FTE & $1,529 a$ & $1,223 b$ \\
\hline FTH & $2,723 a$ & $1,513 b$ \\
\hline FTA & $2,729 a$ & $1,455 b$ \\
\hline FTG & $2,368 a$ & $1,442 b$ \\
\hline Média & 1,963 & 1,330 \\
\hline $\mathrm{CV}$ & 10,44 & 1,38 \\
\hline $\mathrm{R}^{2}$ & 0,89 & 0,98 \\
\hline
\end{tabular}

Valores na mesma linha, seguidos de letras distintas diferem entre si, pelo teste de $F(P<0,05)$

1: $\mathbf{R R}=$ ração-referência, $\mathbf{T I M}=$ trigo integral moído, GT=germe de trigo, FTC = farinha de trigo clara, $\mathrm{FTE}$ = farinha de trigo escura, $\mathrm{FTH}=$ farelo de trigo para uso humano, FTA = farelo de trigo para uso animal, FTG = farelo de trigo grosso.

\section{CONCLUSÕES}

Pelos resultados obtidos neste estudo, conclui-se que: 1- os valores de EMA e EMA $\mathrm{E}_{\mathrm{n}}$ foram afetados pelos níveis de ingestão, inferindo-se que essas medidas não são confiáveis quando obtidas pela metodologia de alimentação forçada; 2 - a EMV apresentou diferenças significativas em quatro alimentos (TIM, FTC, FTE e FTA), inferindo-se que o nível de consumo pode afetar essa medida; 3 - o nível de consumo não afetou a $\mathrm{EMV}_{\mathrm{n}}$, exceto para farinha de trigo clara (FTC), demonstrando que essa medida é a mais adequada quando a EM for avaliada pela metodologia da alimentação forçada ; 4 - a excreção protéica e o balanço de nitrogênio são afetados pelo nível de ingestão, de modo que o menor consumo, pela metodologia da alimentação forçada, pode levar a maiores erros experimentais.

\section{REFERÊNCIAS BIBLIOGRÁFICAS}

ALBINO, L. T. F. Sistemas de avaliação nutricional de alimentos e suas aplicações na formulação de rações para frangos de corte. 1991. $55 \mathrm{f}$. Tese (Doutorado em Zootecnia) - Universidade Federal de Viçosa, Viçosa, 1991.

ALBINO, L. F. T. et al. Utilização de diferentes sistemas de avaliação energéticas dos alimentos na formulação de rações para frangos de corte. Revista da Sociedade Brasileira de Zootecnia, Viçosa, v. 21, p. 10371046, 1992.

CUNNIFF, P. (Ed.). Official methods of analysis of AOAC International. 16. ed. Arlington, 1995. v. 1.

DALE, N.; FULLER, H. L. Correlation of protein content of feedstuffs with the magnitude of nitrogen correction in true metabolizable energy determinations. Poultry Science, London, v. 63, p. 1008-1012, 1984.

DALE, N. M.; FULLER, H. L. Repeatability of true metabolizable energy versus nitrogen corrected true metabolizable energy values. Poultry Science, London, v. 65, p. 352-354, 1986.

DEVEGOWDA, G.; RAMAPPA, B. S.; SHASHIKUMAR, S. K. True metabolizable energy values of some feedstuffs in chickens. Indian Journal Poultry Science, New Delhi, v. 21, p. 2931, 1986. 
FLORES, M. P.; CASTANON, J. I. R. Effect of level of feed input on true metabolizable energy values and their additivity. Poultry Science, London, v. 70, p. 13811385, 1991.

PESTI, G. M. Influence of substitution method and of food intake on bioassays to determine metabolisable energy with chickens. British Poultry Science, London, v. 25, p. 495-504, 1984.

PUPA, J. M. R. et al. Cecectomia em galos sob anestesia local e incisão abdominal. Arquivo Brasileiro de Medicina Veterinária e Zootecnia, Belo Horizonte, v. 50, p. 531-535, 1998.

ROSTAGNO, H. S. et al. Composição de alimentos e exigências nutricionais de aves e suínos. 19. ed. Viçosa: UFV, 1985. 59 p.

SIBBALD, I. R. The effect of level of feed intake on metabolizable energy values measured with adult roosters. Poultry Science, London, v. 54, p. 1990-1997, 1975.

SIBBALD, I. R. A bioassay for true metabolizable energy in feedingstuffs. Poultry Science, London, v. 55, p. 303-308, 1976.
SIBBALD, I. R. The effect of level of feed input on true metabolizable energy values. Poultry Science, London, v. 56, p. 1662-1663, 1977.

SIBBALD, I. R. Effects of level of feed input, dilution of test material, and duration of excreta collection on true metabolizable energy values. Poultry Science, London, v. 58, p. 1325-1329, 1979.

SIBBALD, I. R. The effects dietary cellulose and sand on the combined metabolic plus endogenous energy and amino acid output adult cockerels. Poultry Science, London, v. 59, p. 836-844, 1980.

SIBBALD, I. R.; MORSE, P. M. The effects of level of feed input and excreta collection time on estimates of metabolic plus endogenous energy losses in the bioassay for true metabolizable energy. Poultry Science, London, v. 62, p. 62-68, 1982.

WOLYNETZ, M. S.; SIBBALD, I. R. Relationships between apparent and true metabolizable energy and the effects of a nitrogen correction. Poultry Science, London, v. 63, n. 7, p. 1386-1399, 1984. 